\title{
Research on the coupling traffic system of bus - taxi based on BML
}

\section{model}

\author{
Hui Chu ${ }^{1}$
}

\section{(1. School of Traffic and Transportation, Beijing Jiaotong University, Beijing 100044, China)}

\section{Keywords: Taxicab Bus Joint operation BML model}

Abstract. This article designs a new model based on the BML model. The new model embeds bus elements and passenger elements, and cancels the original operation direction limit and odd/even time step setting. And this paper use control variate method to analyze influence of the number of taxis, the number and position of bus stations and the number of passengers basing on the new model, then collect and analysis data from various combinations, and find the best combination of the utilization of road network of the $\mathrm{L}^{*} \mathrm{~L}$ network. We can summarize the feature of bus-taxi joint operation in the process of comparison and analysis. These features include polymeric complementary public transport priority and Saturation.

\section{Introduction}

The cellular automata model, with its nonlinear behavior and physical characteristics in complex traffic system, has been widely used by many physicists and traffic system to describe some complex phenomena and evolutionary process of traffic flow since the 1990s. Cellular automata generally divides the research object into one-dimensional and two-dimensional models. The typical representative of one dimensional model is the NS model presented by Nagel and Schreckenberg [1]. The main object of this model is the density, speed and flow of traffic flow in freeway and urban Expressway. Typical representatives of two-dimensional model is the BML model presented by Bihan O, Middleton A A and Levine D in 1992 [2]. This model simply and intuitively describes the structural characteristics of urban road network and reveals the process of the evolution of the flow of free flow. Many experts and scholars in China and abroad have carried out many further expansion applications according to these typical models [4]. Fukui and others introduce different vehicle speeds in the BML model. Nagatani studied the asymmetric distribution of vehicles [3]. Xiaoming Liu and others add traffic accidents to the BML model, to analyze the impact of the accident on the traffic status. Jianxun Ding and others embed some typical flyover structures in the model, and analyze the influence of flyover on urban traffic flow. In this paper, the structure of bus stations is embedded in the model, and the concept of passenger land is introduced to study the time that the urban road network recovers from the congestion phase to the free phase.

\section{Taxi-passenger-bus station model}

In the city where the taxi-bus is operated jointly, the main way for residents to travel is by bus. Taxis, as a supplement to bus travel, are generally relatively small capacity in size. In this model, passengers have the initial tendency to move to the bus station, while taxis have the initial tendency to move to the passengers.

On a $\mathrm{L}^{*} \mathrm{~L}$ open grid road network, passengers and taxis randomly appear on the road network model with a given number, instead of different color lattice points. The model sets the initial 
number of passengers and the number of taxis. The model can record the total number of steps taken by the passenger to complete the system (taxis, passengers per move, a unit time), the number of passengers absorbed by the bus station and the number of passengers the taxi carries.

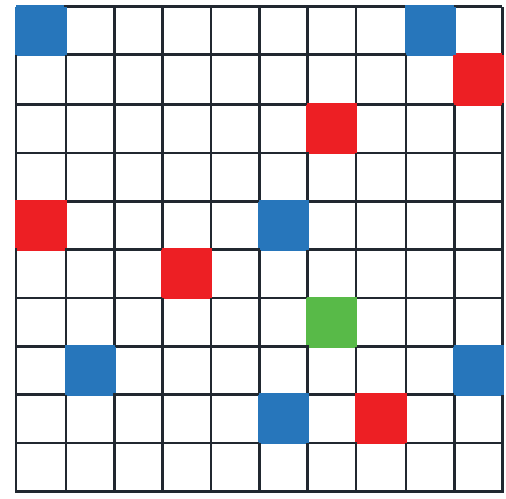

\section{Fig1. The method of presentation of bus stations and taxis}

Fig1 shows a part of a coupled network diagram in which each space represents a space that is occupied by only one passenger or one taxi. The green dot block of the figure represents the bus station, the bus station is set up in the lattice of the $\mathrm{L}^{*} \mathrm{~L}$ and the position of bus station is fixed, do not move with time. The red dot block represents the taxi, which is randomly generated in the grid at the beginning of the model program, and the initial state of taxi distribution is irregular. The blue dot block represents the passenger with the displacement requirement.

Based on the difference between bus and taxi's unit kilometer freight rates, the movement of the model provides for passengers are chased by bus stations and taxis chasing passengers. During the course of a taxi, the passenger is required to run to the nearest passenger and continue to travel a certain distance while catching up with the passengers. In the model, the taxi is given a computing distance, and the distance between the taxi and the passenger is calculated by each time step to find the nearest passenger, to determine the direction of next time the taxi will go. And passengers as a travel individual, the provision of random passengers appear in the road network with the tendency to go to the nearest bus station, each time to calculate the distance of the passengers and the bus station, to determine the next time step to the nearest bus station. The above two modes, because the road network is two-dimensional, when the passengers and taxi is not in the same line, will randomly chooses a direction that can reduce the spacing between them.

\section{Model Termination Condition}

1). Passengers exit the system because they were loaded by bus

When the passenger's coordinates are exactly the same as the bus platform coordinates, it means that they have reached the bus station and entered the bus system, that means the public transport achieved their own travel plans.

2). Passengers exit the system because they were loaded by taxis

When the taxi coordinates are exactly the same as the passenger coordinates, it means that the passengers have been caught by a taxi, that is, the passengers through the taxi to achieve their own travel plans.

\section{3). System Ending}

The beginning of the program operation entered a certain number of passengers, when all passengers have been carried by bus or taxi and exit the system, the entire system runs out. 


\section{Results and Analysis}

In the system where bus and taxi are the main travel modes, the number of buses, the number of taxis and the demand of passengers are the important parameters affecting the whole network. Any one of these changes will cause the entire network accessibility and efficiency. The number of bus stations is bound to make passengers take the bus more convenient and efficient, taxis can affect the number of passengers when they are more active in a certain area and the increase in the number of passenger traffic will inevitably bring enormous and pressure on the entire public transport network. In order to study the relationship between these variables, this paper uses the method of control variables to study the relationship between the three. Coordinate the values of each variable to achieve a more economical, convenient and efficient target.

\section{Passenger aggregation phenomenon}

In real life, passengers in the public transport stations aggregation phenomenon is very obvious. The passengers coming from various places with a variety of different purposes will be near his bus stations gathered. The bus station became an obvious passenger attraction. In the model simulation process, a similar phenomenon occurred. The passengers appearing in different positions will move towards their nearest bus station, because the speed of the bus is certain, so passengers will gather around the bus stations to cause a certain degree of blockage. And when the passengers stop near the bus station, taxis will be attracted by the passengers to reach the outskirts of the bus stations, resulting in a new round of aggregation phenomenon.

As shown on the left side of Figure 2, when the network edge length is 200 and the number of passengers is entered at 1000 , it is clear that passengers gather around several bus stations. Clustered into a cross. This phenomenon is mainly due to the small number of bus stations. All the passengers on the grid need to converge to the only few bus stations, and taxis are much less efficient than the buses, and cannot be completed in time for all passengers.

When the number of bus stations is increased in the model, as shown on the right side of Fig2, stations are added at $(25,25),(25,75),(25,125),(75,25) \ldots(125,125)$ After a total of nine bus stations, comparing the area of the upper left $150 * 150$ size of the whole grid with other regions, it is obvious that the left-grid part of the passenger distribution is more orderly and concentrated in several public transport stations, the number of passengers waiting near the bus station is much less than the left side of Fig2, Congestion in public transport stations has been significantly improved.
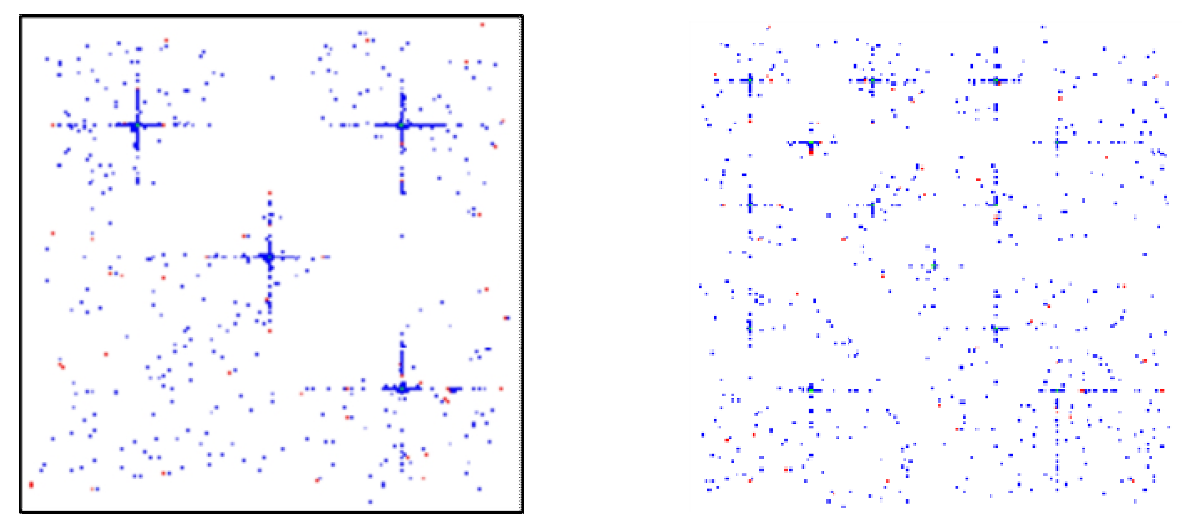

Fig2. Schematic diagram of operation results of different bus stations

By progressively increasing the number of bus stations and entering a passenger number of 100 or 500, you can get the relationship between the total time used of model and the number of bus stations as shown in Fig3. 


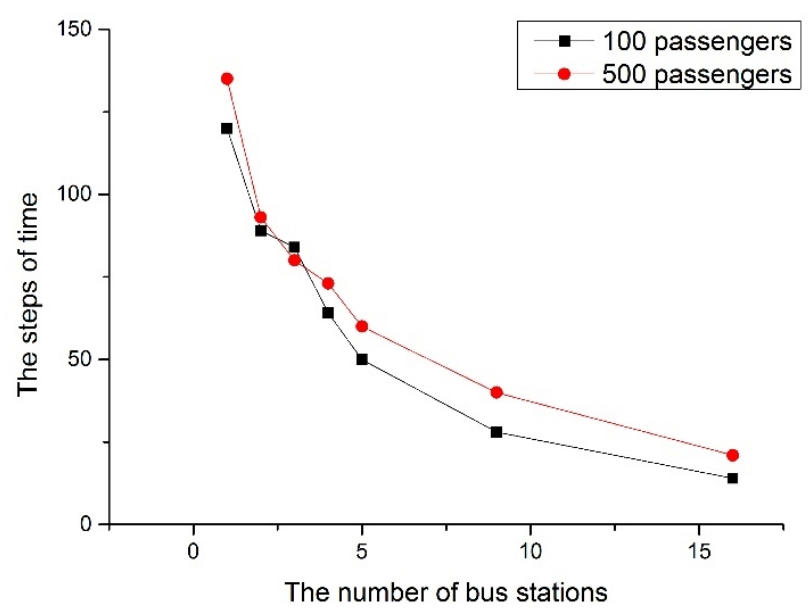

Fig3. The relationship between the use of the model and the number of bus stations

As shown in Fig3, when the number of passengers is 100, the number of bus stations is approaching 1, the time step is very high, the curve is steep. As the number of bus stations increases, the slowing trend from 9 to 16 in Fig3 slows down gradually and eventually tends to a steady state constant. Through the above data can be summed up the number of bus stations and the time of loading passengers approximate to a fixed value.

Taking into account the construction of public transport stations and the convenience of passengers, in the later research, take 5 bus stations for the optimal state for $200 * 200$ Grid network.

The best taxi number based on the taxi carrier number and carrier ratio

Add a new statistic to the model: Taxi number of passengers. Under the condition of the best five bus stations, the paper studies the change of taxi carrier proportion between different passenger numbers and different taxi numbers respectively.

Tab1. The number of taxis and the number of passengers are counted

\begin{tabular}{ccccc}
\hline $\begin{array}{c}\text { Number of } \\
\text { taxis }\end{array}$ & $\begin{array}{c}\text { Taxi carrier } \\
\text { number of 100 } \\
\text { passengers }\end{array}$ & $\begin{array}{c}\text { Taxi carrier } \\
\text { number of 200 } \\
\text { passengers }\end{array}$ & $\begin{array}{c}\text { Taxi carrier } \\
\text { number of 500 } \\
\text { passengers }\end{array}$ & $\begin{array}{c}\text { Taxi carrier } \\
\text { number of 1000 } \\
\text { passengers }\end{array}$ \\
\hline 10 & 5 & 12 & 16 & 27 \\
20 & 12 & 16 & 23 & 40 \\
40 & 18 & 35 & 43 & 75 \\
60 & 26 & 50 & 66 & 104 \\
80 & 34 & 65 & 87 & 130 \\
100 & 42 & 73 & 110 & 150 \\
\hline
\end{tabular}

The results are counted to Tab1, where each of the data is the data obtained from the mean value of 4 simulations. Fig4 is plotted in accordance with Tab1. 


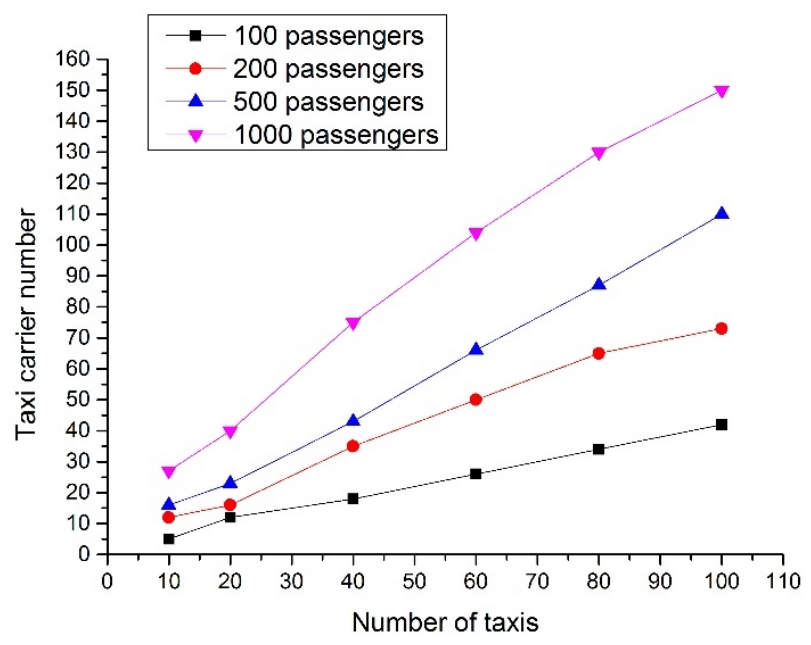

Fig4. Taxi carrier number of different passenger base

Analysis data of Fig4. can found that as the number of passengers gradually increased, the number of passengers in taxis has also increased linearly. At the number of taxis was 10, the number of taxis was only five times times increase while the 10 times-fold increase in passengers from 100 to 1000 . When the model is used to solve the problem, it is found that the number of taxis is random when the number of taxis is very small.

Compared with the increasing number of taxis, it was found that the number of taxi passengers increased faster than the taxi growth rate before taxis reached 60. And after the number of taxis reached 60 , the growth rate of taxi passengers was slower than that of taxi. In the $200 * 200$ grid network, the number of buses in the case of five, the number of taxis in 40 to 60 to obtain the best economic solution.

Taxis in the road network can be carried by taxi number/passenger, as shown in Fig5.

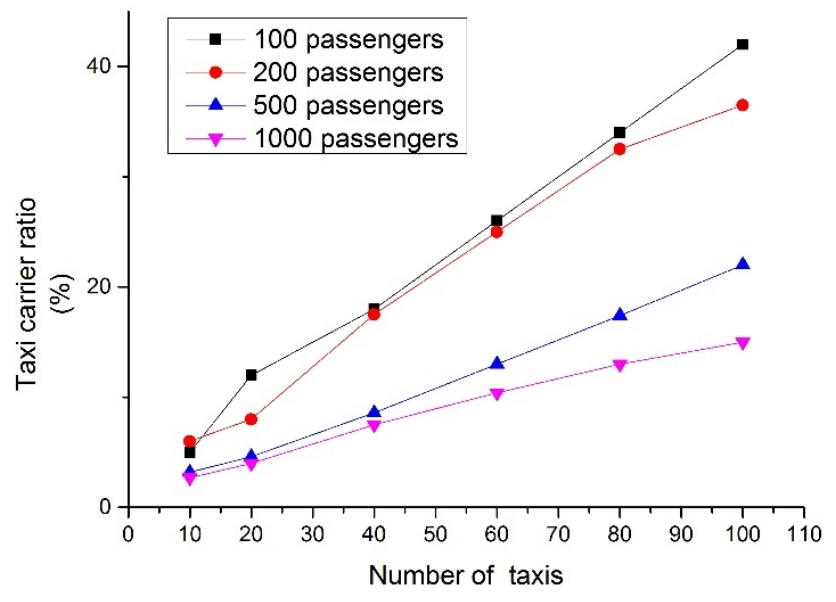

Fig5. Taxi carrier ratio of different passenger base

It can be concluded from Fig5 that as the number of passengers increases, the passenger transport ratio of taxis is gradually reduced. The reason for this situation is that although the number of passengers increased, but due to the number of taxis, the number of carriers is limited, so after the number of passengers reached a certain amount of taxis can only be carried to achieve their own carrier capacity as part of the limit. Compared to Fig4 and Fig5, as the number of passengers increases, the number of passengers carried by taxis will gradually increase, and the proportion of taxis carrying passengers will gradually decrease. When the number of taxis is in the best section 
between 40 60 and the total number of passengers is increased to a certain section of the 200 500, the number of taxi passengers will be close to saturation.

\section{Optimal model and research results}

Combined with the above analysis, the optimal combination of the model can be found at the number of taxis is in the 40 60, the number of bus stations is 5 and the number of passengers in 200 500. We can conclude some characteristics of joint operation of bus and taxi.

1). Aggregation: In taxis and buses have a common source of the premise, passengers tend to converge to the bus site. Taxis, because of the move of the passengers, also produce trends that converge around the bus site.

2). Complementarity: Taxis and buses are complementary. Passengers are less likely to be taken away by taxis when passengers are closer to the bus station. And when the passenger is far from the bus station, because of the long moving distance, the time spent more, will be carried in the process of moving by taxi. This reduces the time that all passengers on the road network have been loaded and improves overall efficiency.

3). Bus Priority: When the number of passengers gradually increased, if the number of bus stations and the total number of taxis will not change, it can be found that the taxi carrier is gradually reduced. On the contrary, the larger the passenger base, the greater the probability of passengers taking the bus.

4). Saturation: The load capacity of taxis will become saturated as the number of passengers increases. According to the model forecast, the bus station will also be saturated and greatly reduce operational efficiency.

\section{Conclusions}

Starting from the thought of BML model, this paper adds the bus station and passengers on the basis of the BML model, and cancels the setting of odd and even time steps, and the running direction is extended from the limited north-south and east-west to the random direction forward mode. A new model is designed based on these settings. By using this model, the numbers of taxis, the number and location of bus stations and the number of passengers are collected to compare and analyze the data in different combinations, and found the optimal combination of the L*L road network. In the process of contrastive analysis, the characteristics of bus-taxi joint operation are summed up. The results can be used in the process of urban planning.

\section{References}

[1] Nagel K,Schreckenberg M.A cellular automaton model for freeway traffic [J]. Journal of Physique. I (France), 1992,2:221-229.

[2] Biham O, Middleton A A, Levine D. Self-organization and a dynamical transition in traffic-flow models[J]. Physical Review A, 1992, 46(10): R6124.

[3] Nagatani T. Effect of jam-avoiding turn on jamming transition in two-dimensional traffic flow model[J]. Journal of the Physical Society of Japan, 1994, 63(4): 1228-1231.

[4] Török J, Kertész J. The green wave model of two-dimensional traffic: Transitions in the flow properties and in the geometry of the traffic jam[J]. Physica A: Statistical Mechanics and its Applications, 1996, 231(4): 515-533. 